\title{
Shift work and risk of cancer in women
}

\begin{abstract}
Despite the benefits of shift work for the general public, the workers themselves are primarily affected by their complications. Many epidemiological studies have mentioned the relationship between work shift and cancer. These studies have focused on the potential negative effects of work-shift jobs, in which the night-light hypothesis and melatonin have received special attentions. This review study details the recent studies and discusses the underlying mechanisms, assumptions and results from epidemiological and laboratory studies on the incidences of cancer in women shift workers. A total of 100 articles were searched, from which 40 were studied. Some recent studies have focused on the relationship between night work shift and breast cancer. The results of the study indicate non-significant correlation between workshift and cancer in general, but particularly, there is a significant relationship between work-shift and breast cancer.
\end{abstract}

Keywords: shift work, risk, cancer, occupational health, women
Volume 8 Issue I - 2019

\author{
Yahya Rasoulzadeha,' Mahdi Hosseinpourib, \\ Vahid Mirzaic,' Omran Ahmadid' \\ 'Department of Occupational Health Engineering, Tabriz \\ University of medical sciences, Iran \\ ${ }^{2}$ Department of Statistics, Persian Gulf University of Bushehr, \\ Iran
}

Correspondence: Omran Ahmadi, Department of

Occupational Engineering, Faculty of Medical Sciences, Tarbiat

Modares University, Tehran, Iran, Tel 09|4424 I240,

Email O.ahmadi@modares.ac.ir

Received: November 09, 2017 | Published: January 22, 2019

\section{Introduction}

Over the last 50 years, the shift working has increased significantly in all developed and developing countries such as Iran. ${ }^{1,2}$ According to the latest statistics, about a quarter of the workforce in the United States is shift working. ${ }^{3,4}$ From a scientific point of view, the definition of shift work is: "employment at work outside of normal working hours, that is, outside the time period ( 7 am to $6 \mathrm{pm}$ )" ${ }^{5,6}$ Despite the usefulness of the shift work for the general public, the workers themselves are mainly affected by the complications. ${ }^{7}$ Lighting the midnight environment causes physiological changes in the secretion of the melatonin hormone. ${ }^{8}$ In a number of epidemiological studies focusing on the relationship between shift work and cancer, attention was focused on the potential negative effects of shift work, with the special emphasis on nighttime light and melatonin. In these studies, there has been an inverse relationship between continuous exposure to light at night and melatonin level. ${ }^{8,9}$ This reduction in melatonin production is detailed in a hypothesis that light exposure results in an increase in the production and amount of hormones such as estrogen, as well as changes in the growth trend of hormones Tumors in the chest. ${ }^{9,10}$ According to the results of objective studies, the risk of lung and breast cancers is high in individuals who worked as shift workers. ${ }^{11}$ The results of laboratory studies and animal studies have shown that the effects of low production of melatonin are not limited to breast cancer. ${ }^{12,13}$ This review study details the recent systematically studies, and discusses the underlying mechanisms, assumptions, and results from epidemiological and laboratory studies on the incidences of cancer in women shift workers.

\section{Method}

This is a review article on shift working and its relation to women cancer. The results of the study include the results of recent papers on shift working and its association with cancer as well as chronic diseases, which were worked out as a review of studies by July 2016 . To search for the desired articles from the combination of cancer keywords, shift work, the chance of cancer in PubMed, Google Scholar and science direct A total of 100 articles were searched with the keywords mentioned, from which 40 articles related to this topic where chosen. The main results of the papers were summarized and are explained in greater detail. Articles include epidemiological studies of groups that work continuously as shift workers, people who work on a daily basis or often work at night rotational and people work for daily. In this overview, the term "shifts work" was used to compare any type of job that was characterized as an abnormal and irregular working hours with people who regularly work for daily.

\section{Results}

Recent studies have focused on the relationship between night work shift and breast cancer. Details of studies on shift work and the risk of developing cancers are presented. In the study of IARC groups, out of eight studies, six with long-term night shifts were shown to have an increase in the risk of cancer compared to those who did not work at night. ${ }^{14}$ In the study of night work and the risk of breast cancer among Norwegian nurses, there was a strong correlation between increased risk of cancer in nurses working for more than 5 years with 6 night shift per month $(\mathrm{OR}=1.8)$. There was no significant relationship between the duration of work and the risk of incidence of cancer. ${ }^{15}$ Also, in the cohort study by Pronk. on Chinese women, there was no significant increase in the duration of work shift and the risk of cancer ${ }^{16}$ Knutsson and colleagues studied breast cancer among shift workers in a cohort study. For people working during day time OR was 1.3 while for those with a night shift it was 2.02 , suggesting an increase in the risk of developing cancer among women shifting overnight. ${ }^{17}$ In a study by Megdal. ${ }^{17}$ who conducted a systematic study and meta-analysis of 13 types of studies in 2005, in seven studies the relationship between breast cancer and the night shift was indicated with $R R=1.48$. The results of this study showed that there is a relationship between long-term overnight shift and increased risk of developing breast cancer. ${ }^{18}$ Peplonska in his study on nurses showed that working 8 times or more in the form of night shifts per month can interfere with the synthesis of melatonin. ${ }^{19}$ Anne Grundy showed that there is a relationship between long-term night shift with increased risk of breast cancer. ${ }^{15}$

Kolstad conducted a systematic review of the relationship between shift work nighttime and breast cancer and other cancers indicated that there is no evidence related to night shift work and the risk of developing cancer in different parts of the body. ${ }^{20}$ Hansen in a casecontrol study, showed an increase in the risk of cancer among women who work mainly at night (RR 1.5 in men and 1.7 in women). ${ }^{21}$ Davis 
et al worked on the risk of breast cancer and shift work and showed an increase in the risk of cancer among women (RR 1.06 in men and 1.6 in women). ${ }^{22}$ The cohort study of Schernhammer on shift work and risk of breast cancer in women showed an increase in the risk of cancer among women (RR 1.08 in men and1.36 in women). ${ }^{23}$

\section{Conclusion}

Shift workers are faced with a variety of risk factors that affect the risk of cancer. Increased exposure to light at night is believed to be one of the probable causes of breast cancer in the industrialized world. The nighttime brightness may affect the natural secretion of melatonin and more likely to be secreted. Suppressing the production of melatonin may be a risk factor for breast cancer by direct and indirect mechanisms, such as dangerous changes in the concentration of sex hormones. This epidemiological review details a relationship between shift work and the risk of cancer. There are definite evidences for the relationship between shift work and the risk of breast cancer, but there is limited evidence of the relationship between night work and cancer in other parts of the body. A number of mechanisms may form such relationship, including affecting the production of melatonin, sleep disturbances and lifestyle. In most studies, disturbances in circadian cycles under the influence of night lighting are one of the higher risk factors for cancer than other factors, such as the physical indices of night workers or the smoking habits of night workers. The results of epidemiological researches should be used to develop the information of work regulations and public health interventions and reduce the risk among shift workers. In addition, the duration and frequency of exposure, working history and demographic characteristics and other factors may influence the incidence of cancer.

\section{Acknowledgments}

None.

\section{Conflicts of interest}

The author declares there are no conflicts of interest.

\section{References}

1. Bazazan A, Rasoulzadeh Y, Dianat I, et al. Demographic Factors and their Relation to Fatigue and Mental Disorders in 12-Hour Petrochemical Shift Workers. Health Promotion Perspectives. 2014;4(2):165-172.

2. Rasoulzadeh Y, Bazazan A, Safaiyan A, et al. Fatigue and Psychological Distress: A Case Study Among Shift Workers of an Iranian Petrochemical Plant, During 2013, in Bushehr. Iranian Red Crescent Medical Journal. 2015;17(10):e28021.

3. Costa G. Shift work and occupational Medicine: an overview. Occup Med (Lond). 2003;53(2):83-88.

4. Knutsson A. Health Disorders of shift workers. Occup Med (Lond). 2003;53:103-108.

5. Siegel JM. Clues to the functions of mammalian sleep. Nature. 2005;437(7063):1264-1271.
6. Saberi H, Moraveji A, Parastouie K. Metabolic syndrome among professional bus and truck drivers in Kashan. ISMJ. 2008:12(2):126-132.

7. Folkard S, Tucker P. Shift work, safety and productivity. Occup Med (Lond). 2003;53(2):95-101.

8. Zeitzer JM, Dijk DJ, Kronauer R, et al. Sensitivity of the human circadian pacemaker to nocturnal light: melatonin phase resetting and suppression. J Physiol. 2000;526:695-702.

9. Czeisler CA, Dijk DJ. Chapter 20: human circadian physiology and sleep-wake regulation. In: Takahashi JS, Turek FW, Moore RY, editors. Handbook of behavioral neurobiology: circadian clocks. New York (NY): Plenum Publishing; 2001. 531 p.

10. Graham C, Cook MR. Examination of the melatonin hypothesis in women exposed at night to EMF or bright light. Environ Health Perspect. 2001;109(5):501-507.

11. Rafnsson V, Tulinius H, Jonasson JG, et al. Risk of breast cancer in femal flight attendants: a population-based study (Iceland). Cancer Causes Control. 2001;12(2):95-101.

12. Hill SM, Blask DE. Effects of the pineal hormone melatonin on the proliferation and morphological characteristics of human breast cancer cells (MCF-7) in culture. Cancer Res. 1988; 48(21):6121-6126.

13. Cos S, Mediavilla MD, Fernandez R, et al. Does melatonin induce apoptosis in MCF-7 human breast cancer cells in vitro? J Pineal Res. 2002;32(2):90-96.

14. Straif K, Baan R, Grosse Y et al. Carcinogenicity of shiftwork painting, and fire-fighting. Lancet Oncol. 2007;8(12):1065-1066.

15. Jenny-Anne S. L ، Night Work and Breast Cancer Risk among Norwegian Nurses: Assessment by Different Exposure Metrics. American Journal of Epidemiology. 2011;173(11):1272-1279.

16. Pronk A, Ji BT, Shu XO, et al. Night-shift work and breastcancer risk in a cohort of Chinese women. Am J Epidemiol. 2010;171(9):953-959.

17. Knutsson A. Shift work and coronary heart disease. Scand J Soc Med. 1989;44(Suppl.):1-36.

18. Megdal SP, Kroenke CH, Laden F, eta 1. Nightwork and breast cancer risk: a systematic review and meta-analysis. Eur $J$ Cancer. 2005; 41(13):2023-2032

19. Peplonska B. Night shift work characteristics and 6-sulfatoxymelatonin (MT6s) in rotating night shift nurses and midwives. Occup Environ Med. 2014;69(5):339-346.

20. Kolstad HA. Nightshift work and risk of breast cancer and other cancers-A critical review of the epidemiologic evidence. Scand J Work Environ Health. 2008;34(1):5-22.

21. Hansen J. Increased breast cancer risk among women who work predominantly at night. Epidemiology. 2001;12(1):74-77.

22. Davis S, Mirick DK, Stevens RG. Night shift work, light at night, and risk of breast cancer. J Natl Cancer Inst. 2001;93(20):1557-1562.

23. Schernhammer ES, Laden F, Speizer FE, et al. Rotating night shifts and risk of breast cancer in women participating in the Nurses' Health Study. J Natl Cancer Inst. 2001;93(20):1563-1568. 